\title{
Local structural study of novel mott-insulating cousins of the iron pnictides
}

\author{
B. Karki ${ }^{1}$, A. Alfailakawi ${ }^{1}$, B. A. Frandsen ${ }^{2}$, J. C. Neuefeind ${ }^{3}$, M. Everett ${ }^{3}$ and B. Freelon ${ }^{1}$ \\ ${ }^{1}$ Department of Physics and Astronomy, University of Louisville, Louisville, KY 40208, USA, \\ bmkark01@louisville.edu,alaa.alfailakawi@louisville.edu,bkkfree02@louisville.edu, \\ 2 Department of Physics and Astronomy, Brigham Young University, Provo, UT 84602, USA, \\ benfrandsen@gmail.com \\ ${ }^{3}$ Oak Ridge National Laboratory, Oak Ridge, TN 37831, USA, \\ neuefeindjc@ornl.gov,everettsm@ornl.gov
}

\begin{abstract}
:
There has been significant interest in mixed anion materials since the discovery of high transition temperature $\left(T_{\mathrm{c}}\right)$ superconductivity in layered iron pnictides system. Electron-electron interaction leads to the emergence of fascinating collective electronic behavior, including novel magnetism, electronic nematic order and superconductivity. All of these properties are closely related in iron-based superconductors. The interplay between these orders can be understood by studying their co-evolution across the phase diagram through the chemical substitution. An important question in iron-based superconductivity has been whether superconductivity could emerge from materials that are in the Mott insulating region of the electronic phase diagram as is the case for cuprate high- $T_{\mathrm{c}}$ superconductors. In an effort to find such materials, the mixedanion iron-oxychalcogenides have been explored.
\end{abstract}

Iron oxy-chalcogenides, $\mathrm{La}_{2} \mathrm{O}_{2} \mathrm{Fe}_{2} \mathrm{OM}_{2}(M=\mathrm{S}, \mathrm{Se})$, is the layered materials formed from stacking layered units of $\mathrm{La}_{2} \mathrm{O}_{2}$ and $\mathrm{Fe}_{2} \mathrm{OM}_{2}(M=\mathrm{S}, \mathrm{Se})$. They are structurally reminiscent of the cuprates while possessing the Fe-chalcogen planes that are similar to the Fe-pnictides. This work describes the structural properties of, $\mathrm{La}_{2} \mathrm{O}_{2} \mathrm{Fe}_{2} \mathrm{OM}_{2}(M=\mathrm{S}, \mathrm{Se})$ which are closely related to the superconductivity because they are on the verge of a metal insulator transition. By exchanging $\mathrm{S}$ and Se it is possible to tune the electron correlation value from insulating to the metallic phase. Unlike the Fe-pnictides these materials do not show long range structural phase transition. However, both materials $\mathrm{M}=\mathrm{S}$, Se undergo magnetic phase transitions from paramagnetic antiferromagnetic phase at a Neel temperature $107 \mathrm{~K}$ and $90 \mathrm{~K}$, respectively.

Neutron total scattering experiments on $\mathrm{La}_{2} \mathrm{O}_{2} \mathrm{Fe}_{2} \mathrm{OM}_{2}(M=\mathrm{S}$, Se) were performed on Nanoscale Ordered Materials Diffractometer (NOMAD) beamline at Oak Ridge National Laboratory (ORNL) in order to collect Pair distribution function (PDF) data. PDF analysis involves Fourier transforming the measured total scattering intensity in order to obtain a real space representation of inter-atomic correlations. This technique is used to study local, short range structural correlations that deviate from the average structure. Our results for $M=\mathrm{S}$, Se possess short-scale structural distortions however, neutron powder diffraction (NPD) provides clear evidence that the average, long-range structure remains tetragonal throughout the high and low temperature regimes. Local crystal structure was studied by investigating deviations in atomic positions and orthorhombicity. By tracking the orthorhombicity parameter we observe local scale distortions between the tetragonal and orthorhombic structure in a typical range of 1-2 nm. These spatially limited distortions represent fluctuating nematic order which suggests the ubiquity of nematic fluctuations in iron-based superconductors and related materials. In addition, we found the discontinuity in $c$-lattice which may arise due to the buckling of $\mathrm{Fe}_{2} \mathrm{O}$ plane. We anticipate that this buckling might be due to the change in octahedral height or tilting of the octahedral structure. Overall, these results suggest that structural distortion may play a role in absence of superconductivity in these materials. 